\title{
Repurposing of thalidomide and its derivatives for the treatment of SARS-coV-2 infections: Hints on molecular action
}

\author{
Lakshmikirupa Sundaresan $^{1}$, Suvendu Giri ${ }^{2}$, Himanshi Singh ${ }^{2}$, and Suvro Chatterjee ${ }^{2}$ \\ ${ }^{1}$ Hospital for Sick Children \\ ${ }^{2}$ AU-KBC Research Centre
}

June 5, 2020

\begin{abstract}
Background and Purpose: SARS-coV-2 pandemic continues to cause an unprecedented global destabilization. There is an urgent need to develop vaccines or identify molecules to treat severe cases and repurposing of drugs is the best approach at this hour. Thalidomide, despite having an infamous history has been successfully repurposed and tested for various disease conditions including inflammatory diseases and tumor. Few reports emphasize the use of thalidomide with a SARS-coV-2 pneumonia patient being successfully treated with thalidomide. Experimental Approach: A meta-analysis comparing the transcriptomes of SARS-coV-2 infected tissues with thalidomide and lenalidomide-induced transcriptomic changes in transformed lung, endothelial and hematopoietic models was performed. Key Results: Thalidomide and lenalidomide exhibited pleiotropic effects affecting a range of biological processes including inflammation, immune response, angiogenesis, MAPK signaling, NOD-like receptor signaling, TLR signaling, leukocyte differentiation and innate immunity, the processes which are aberrantly regulated in severe COVID-19 patients. In addition, we show the similarities between the expression profiles of SARS-coV-2 infected lung and systemic lupus erythematous. Conclusion and Implications: The present study recommends thalidomide analogs as a "better fit" to treat severe cases of novel viral infections, healing the damaged network by compensating the impairment caused by the Coronavirus disease-2019 (COVID-19).
\end{abstract}

Repurposing of thalidomide and its derivatives for the treatment of SARS-coV-2 infections: Hints on molecular action

Running title: Possible mechanisms of thalidomide in treating COVID-19

Lakshmikirupa Sunderesan $^{¥}$, Suvendu Giri\#,* , Himanshi Singh\#,*, and Suvro Chatterjee\#,*

*Vascular Biology Laboratory, AU-KBC Research Centre, Chennai, India.

\# Department of Biotechnology, Anna University, Chennai, India.

${ }^{¥}$ Developmental \& Stem Cell Biology Program, The Hospital for Sick Children, Toronto, ON M5G 1X8, Canada

\section{Correspondence:}

Suvro Chatterjee Ph.D,

AU-KBC Research Centre,

MIT Campus of Anna University,

Chromepet, Chennai-600044, India.

Tel.: +91442223 4885×48/+91442223 2711×48; 
Fax: +91442223 1034

E-mail address: soovro@yahoo.ca

Word count : 2,733

Acknowledgement : This project was partially supported by a grant from University Grant CommissionFaculty Recharge Programme (UGC-FRP), Government of India to SC.

Conflict of Interest : The authors declare that there are none.

Abstract

Background and Purpose : SARS-coV-2 pandemic continues to cause an unprecedented global destabilization. There is an urgent need to develop vaccines or identify molecules to treat severe cases and repurposing of drugs is the best approach at this hour. Thalidomide, despite having an infamous history has been successfully repurposed and tested for various disease conditions including inflammatory diseases and tumor. Few reports emphasize the use of thalidomide with a SARS-coV-2 pneumonia patient being successfully treated with thalidomide.

Experimental Approach: A meta-analysis comparing the transcriptomes of SARS-coV-2 infected tissues with thalidomide and lenalidomide-induced transcriptomic changes in transformed lung, endothelial and hematopoietic models was performed.

Key Results: Thalidomide and lenalidomide exhibited pleiotropic effects affecting a range of biological processes including inflammation, immune response, angiogenesis, MAPK signaling, NOD-like receptor signaling, TLR signaling, leukocyte differentiation and innate immunity, the processes which are aberrantly regulated in severe COVID-19 patients. In addition, we show the similarities between the expression profiles of SARS-coV-2 infected lung and systemic lupus erythematous.

Conclusion and Implications: The present study recommends thalidomide analogs as a "better fit" to treat severe cases of novel viral infections, healing the damaged network by compensating the impairment caused by the Coronavirus disease-2019 (COVID-19).

Keywords: SARS-coV-2, Thalidomide, Lenalidomide, CC-220, Pomalidomide, Inflammation, Immune Response, Angiogenesis

Abbreviations : COVID-19 - Coronavirus Disease -19; BALF - Broncho-alveolar lavage fluid; PBMC Peripheral Blood Mononuclear Cell; DEG- Differentially Expressed Genes; GSEA - Gene Set Enrichment Analysis; SLE - Systemic Lupus Erythematosus; TLR - Toll-like Receptor; HUVEC - Human Umbilical Vein Endothelial Cell

\section{Introduction}

Novel coronavirus, SARS-coV-2 has been posing devastating effects on a global scale with a soaring number of infections and an alarming rate of mortality. Despite tremendous efforts, development of effective vaccines or anti-viral medications is months away leaving repurposing of drugs with known safety and efficacy profiles as the only viable choice. COVID-19 is clinically very challenging since the novel coronavirus triggers multiorgan turbulence devastating the homeostasis of the human system. Once the SARS-CoV-2 virus enters the respiratory tract, there are four different stages of the infection from symptoms to multi-organ failures. Phase I starts with the ACE2 receptor-mediated viral entry through nasal and oral routes followed by host immune system alert simultaneously with active viral replication in the upper respiratory tract (Phase II). Next, Phase III coincides with minor cytokine storm in the alveoli releasing the inflammatory cytokines making the blood vessels leaky around the air sac, and ultimately the second cytokine storm arrives with uncontrolled inflammatory and life-threatening symptoms, acute respiratory distress syndrome (ARDS), seizure, severe hypoxia and severed organ toxicity (Phase IV). Manifestation of the bi-phasic cytokine storm occurs through the activation of a series of cytokines including granulocyte-colony stimulating factor (G-CSF), interferon gamma-induced protein 10 (CXCL10), monocyte chemoattractant protein 1 (MCP1), 
macrophage inflammatory protein $1 \alpha$ (MIP-1 $\alpha)$, tumour necrosis factor $\alpha$ (TNF- $\alpha$ ), interleukin (IL)-2R and IL-6 overwhelming the system leading to indiscriminate damages in multiple organs. There is an increased amount of blood vessel growth in the lungs of COVID-19 patients compared to severe influenza . Due to such multi-layers of problems associated with the COVID-19 infections, researchers around the world are desperately in search for a drug, which would able to tackle all or few of these COVID-19 hallmarks.

Thalidomide, a small molecule drug, was marketed with an intention to relieve morning sickness in pregnant women, and took a wrong turn that resulted in children with birth defects upon in-utero exposure . Later, thalidomide became a game changer for its the multi-faced pharmacological effects such as immunomodulation, anti-inflammation, anti-angiogenesis, and anti-viral effects . At this point of time, the world needs a "smart" solution. Therefore, thalidomide raises the hope for treating COVID-19 patients Chen et al reports successful treatment of SARS-coV-2 associated pneumonia with combinatory treatment of thalidomide and a low-dose glucocorticoid Two clinical trials, NCT04273581 and NCT04273529 have been registered to check the efficacy of thalidomide in treating COVID-19 patients. The adverse effects of thalidomide and its analogs are well documented. Various genes aberrantly expressed in SARS-coV-2 affected lungs are known targets of thalidomide (Table S1). Extensive information available on thalidomide's mechanisms, their efficacy and safety in hemophagocytic syndrome-induced cytokine storm and idiopathic pulmonary fibrosis, severe H1N1 and paraquat poisoning lung injury argue for the possible action of thalidomide on COVID-19 induced lung effects and cytokine storm.

Transcriptome-based approach to connect diseases with drug responses is a recognized strategy in drug repurposing . With the fast-growing literature on SARS-coV-2 infections, we performed meta-analysis of whole transcriptome signatures of lungs, PBMC, BALF from SARS-coV-2 affected patients and A549 cells and compared with the gene expression signatures of thalidomide or lenalidomide-treated A549 (transformed adenocarcinoma cells), hematopoietic and endothelial cells. We hereby provide possible mechanistic actions of thalidomide in treating the SARS-coV-2 pathology. In addition, we suggest that the derivatives of thalidomide, lenalidomide and CC-220 might also be effective in the treatment of SARS-coV-2.

\section{Methods}

\section{Data Collection}

A total of 16 gene expression datasets including 15 publicly available expression datasets were meta-analyzed in this study. Transcriptomes of SARS-coV-2 infected lung tissues matched with healthy control and SARScoV-2 treated A549 cells were obtained from GEO (Accession ID GSE147507). Transcriptome data of bronchoalveolar lavage fluid (BALF) and peripheral blood mononuclear cells (PBMC) were obtained from the recently published study . Differentially expressed genes (DEG) from the expression profiles of systemic lupus erythematosus (Accession ID: GSE112087), bone marrow cells treated with lenalidomide (Accession ID: GSE106748), lymphoma cells treated with lenalidomide (Accession ID: GSE60618), CD34 positive cells treated with pomalidomide (Accession ID: GSE144052), MERS infected PBMC (Accession: GSE1739), lenalidomide treated PBMC (Accession ID: GSE84251) and CC-122 treated lymphoma cells (Accession ID: GSE75420) were obtained using limma from the GEO database. Library of Integrated Network-Based Cellular Signatures (iLINCS) is a database which contains the gene expression signatures of more than 21,000 compounds (http://www.ilincs.org/ilincs/). We obtained the gene expression signatures for A549 cells treated with $10 \mu \mathrm{M}$ thalidomide for $6 \mathrm{~h}$ (LINCSCP_4683) and 24h (LINCSCP_4463), $100 \mu \mathrm{M}$ lenalidomide for 6h (LINCSCP_4650) and 24h (LINCSCP_4427).

\section{Transcriptome sequencing}

SARS-coV-2 affects the cardiovascular system to a great extent and to understand the effects of thalidomide and its derivatives, human umbilical vein endothelial cells (HUVEC) were subjected to $20 \mu \mathrm{M}$ thalidomide or $20 \mu \mathrm{M}$ lenalidomide or $20 \mu \mathrm{M}$ pomalidomide or vehicle control treatment for $8 \mathrm{~h}$. RNA was isolated using TRIzol@ method and whole transcriptome sequencing was performed using Illumina HiSeq 2500 platform. The data can be accessed at Gene Expression Omnibus (GEO) with the Accession ID: GSE118979. The sequence reads were aligned with reference genome of Homo sapiens using TopHat2 (v2.0.8) and the followed 
by transcript compilation and gene identification was done using Cufflinks (v2.2.0) . The DEG were identified using Cuffdiff program (v2.2.0)

\section{Differential Expression and Enrichment Analysis}

The raw counts from the SARS-coV-2 transcriptomic profiles were subjected to differential expression analysis by DESEq2 v1.26.0 . Subsequently the genes were pre-ranked using the p-values from DESeq2 analysis and subjected to pre-ranked gene set enrichment analysis (GSEA) . Gene sets with FDR $<0.05$ were considered to be statistically significant and were visualized using EnrichmentMap plugin of Cytoscape . For drug signatures from iLINCS, differentially expressed genes with $\mathrm{p}<0.05$ were considered to be statistically significant. Enrichment of kinase perturbation was carried out using Enrichr .

\section{KINOMEscan Kinase Screening}

We analyzed our previously published KINOMEscan kinase screening dataset of thalidomide in order to investigate how thalidomide affects the immune system. Kinases whose activities were reduced at least by $60 \%$ were considered for further enrichment analysis.

\section{Comparative analysis using Toppcluster}

The differentially expressed genes $(\mathrm{Q}<0.05$ for transcriptome and $\mathrm{p}<0.05$ for drug signatures) from all the gene expression profiles were compared for overlapping genes and over-represented pathways using Toppcluster and the networks were visualized using in Cytoscape .

\section{Identification of protein targets using PharmMapper}

For identifying the protein targets of the thalidomide, we utilized the PharmMapper server (http://www.lilab-ecust.cn/pharmmapper/) . The server identifies possible physiological protein targets of any drug molecule by using a pharmacophore-based mapping approach. The three-dimensional structure of thalidomide was obtained from PubChem and processed on the PharmMapper server choosing only human protein target sets. The top 100 target proteins were selected based on the ranking associated with a fit score (pKd value) for further enrichment analysis using Enrichr .

\section{Results}

Meta-analysis of SARS-coV-2 affected lung biopsies and PBMCs reveal enrichment of various pathways pertaining to immune response

SARS-coV-2 infection caused a massive surge in inflammatory response, cytokine production and cytokinemediated signaling. There was a substantial up-regulation of immune response including the processes of hematopoietic development and lymphocyte activation (Figure 1A,3A,3B). Activation of viral life cycle and anti-viral interferon signaling was observed in infected lungs and A549 cells (Figure 1B, 5). Overrepresentation of pathways including NOD-like receptor signaling, MAPK cascade, Measles and InfluenzaA were seen in the infected lung as identified by gene set enrichment analysis (Figure 1A). Targets of transcription factors, ISRE and IRF were upregulated in SARS-coV-2 affected lungs similar to up-regulation observed in the PBMC of SLE patients (Figure 1B, 1C). Enrichr analysis revealed that the genes up-regulated in SARS-coV-2 lung were down-regulated when SYK was knocked down or inhibited (Figure 1D). Expression to kinase (X2K) analysis showed the possible perturbation of various MAP kinases, ABL1 and JNK1 (Figure $1 \mathrm{E}, 1 \mathrm{~F})$. Human phenotype enrichment analysis shows thrombocytopenia, poor wound healing, abnormality of lymphatic system, serositis and abnormal anticoagulant pathways in SARS-coV-2 infected lungs (Figure $\mathrm{S} 1)$.

\section{Effect of Thalidomide on Kinases Implicated in Immune Response and MAPK signaling}

The kinase screening assay identified key kinases involved in the regulation of immune response are functionally modulated by thalidomide. The most kinase affected was LCK and SYK (Figure 2C), critical modulators of T cell receptor signaling. Many LCK substrates, SPI1, TBK1, FOXP3, EGR1, ESR1, IRF1, CBL and STAT1 as well as SYK phosphorylation targets such as OAS1 and MX1 were up-regulated in SARS-coV-2 
lung. Various processes mediating immune response including JUN phosphorylation, IKappaB phosphorylation, JAK-STAT pathway, leukocyte mediated immunity, neutrophil degranulation and activation, B cell receptor signaling and MAPK cascade were found to be affected by thalidomide (Figure 2D). PharmMapper results showed strong affinity for LCK, HCK and SYK along with other proteins involved in innate and adaptive immune response (Table S2, S3, S4). We studied the effects of thalidomide and its derivatives on endothelium and identified the down-regulation of several angiogenic genes (Figure S5 and Table S1).

\section{Up-regulated pathways and GO Biological Processes in SARS-coV-2 infection suppressed by thalidomide and lenalidomide}

SARS-coV-2 infected lungs, PBMC and A549 cells showed significant upregulation of expression of genes involved in inflammation, cytokine signaling, MAPK signaling and activation of cells mediating the immune response whereas BALF exhibited a slightly different immune profile where were leukocyte and neutrophil activation was suppressed (Figure 3A). Comparison of differentially expressed genes of all the signatures yielded interesting results. Many of the processes up-regulated in SARS-coV-2 infected tissues were suppressed by thalidomide and lenalidomide in A549 cells and endothelial cells (Figure 2A,2B,3A). Thalidomide-treated A549 cells showed suppression of key genes including SYK, JUN, PIK3CA and HLA genes implicated in immune response (Figure 3B, 3C). Thalidomide and lenalidomide down-regulated various pro-inflammatory and angiogenic genes aberrantly expressed in SARS-cov-2 infected lungs including CCL2 and TSC22D3 which are NF- $x$ B modulators in A549 cells (Figure S3, S4). Thalidomide and lenalidomide treatment resulted in significantly suppression of cytokine response, angiogenesis, inflammation, Fc Epsilon receptor signaling and MAPK cascade (Figure 2A). In addition, lenalidomide down-regulated STAT1 expression, leukocyte differentiation, TLR signaling as well as IRF activation (Figure 2B, 4B). Many genes implicated in NOD-like receptor signaling overexpressed in SARS-coV-2 were suppressed by lenalidomide in A549 and lymphoma cells (Figure 4C). B cell receptor signaling was activated in SARS-coV-2 affected PBMC whereas $\mathrm{T}$ cell activation was observed in SARS-coV-2 lungs. Translation of viral mRNA was exclusively observed in SARS-coV-2 infected BALF whereas genes implicated in viral entry and life cycle were up-regulated in SARS-coV-2 infected lungs, BALF and A549 cells. Genes involved in viral entry and type I interferon signaling were down-regulated in thalidomide-treated A549 cells and lenalidomide-treated lymphoma, A549 and HUVEC (Figure 5A, 5C).

\section{Similarity of SARS-coV-2 expression profile with that of SLE, lymphoma and multiple myeloma}

Comparative enrichment analysis of gene expression profiles for disease-specific phenotypes revealed the similarity of SARS-infected tissues with pneumonia, influenza, lymphoma, systemic lupus erythematosus (SLE), multiple myeloma, asthma, auto-immune diseases, asthma, pneumonia, and atherosclerosis while SARS-coV-2 PBMC showed exclusive overlap with lymphoma, multiple myeloma and chronic lymphocytic leukemia (CLL) (Figure 6A). The gene expression profile of SARS-coV-2 infected lungs was very similar to that of PBMC from SLE patients (Figure 6C). There was a significant overlap between SARS-coV-2 and SLE in up-regulated genes involved in immune regulation (Figure 3B), interferon signaling (Figure 5B) and disease-specific phenotypes (Figure 6B, 6D, 6E). Many genes deregulated in lymphoma and multiple myeloma were up-regulated in SARS-coV-2 infections as well (Figure 6B, 6E). Several aberrantly expressed genes involved in these disease phenotypes were down-regulated in thalidomide and lenalidomide treated A549 and endothelium (Figure 6).

\section{Discussion}

SARS-coV-2 infection causes surge in a number of pathways related to inflammation, cytokine signaling, leukocyte and lymphocyte activation, innate and adaptive immune response marking the phenomenon of "cytokine storm". As the whole immune system is affected during the SARS-coV-2 infection, immunomodulators would be highly beneficial in treating the symptoms. A COVID-19 patient with pneumonia was treated successfully with thalidomide and low dose glucocorticoid. There was a significant decrease in the inflammatory cytokines including IL1-, IL-6 and IFN- and increase in the CD4+ and CD8+ T cells and NK

cells. Thalidomide reduced the severity of many COVID-19 symptoms such as lung lesions, exudation due 
to its pleiotropic effects on the human system . Hemophagocytic syndrome, a hyperinflammatory disorder is also another condition in which cytokine storm occurs. It is frequently present with extranodal natural killer/T cell lymphoma (ENKTL). Thalidomide was effective in suppressing the cytokine storm through inhibition of NF- $x$ B based transcription of IFN- and TNF genes and thalidomide along with P-Gemox was highly effective in treating ENKTL patients in a Phase II clinical trial . Comparison of SARS-coV-2 expression profiles with drug signatures through enrichment analysis revealed striking actions of thalidomide and lenalidomide in A549 and endothelial cells. The results suggest that thalidomide and lenalidomide could reverse the devastating effects of SARS-coV-2 infections on immune system. We selected A549, an adenocarcinomic human alveolar basal epithelial cell line to test our hypothesis that thalidomide would be effective against the cytokine storms. The A549 cell line is an appropriate model for testing cytokine storm targeting drugs since a previous study established this model by infecting the cells with influenza A/H1N1 virus (PR-8) or nonstructural protein 1 (NS1) plasmid to test the mechanisms behind inflammatory cytokines/chemokines mediated "cytokine storm" Studies have utilized A549 cells to show the effects of thalidomide on lung fibrosis . A limitation of this study is that only 978 genes called "landmark genes" are profiled in the iLINCS drug signatures. However, the profiles are highly reproducible and represent the whole transcriptome . Our models of A549 and HUVEC effectively capture the effects of thalidomide in lungs as well as endothelium.

It is also emerging that SARS-coV-2 infections perturb vascular plexus significantly and there is a substantial increase in the growth of new blood vessels and evidence of intussusceptive angiogenesis with overexpression of angiogenesis and hypoxia genes in the lungs of COVID-19 patients. Cytokine storm and atherosclerosis are tightly connected in SARS-coV-2 which is consistent with our analysis revealing the enrichment of atherosclerosis in the SARS-coV-2 signatures (Figure 6). Thalidomide is a renowned modulator of vascular system, and it is known to transcriptionally or functionally target various genes (Table S1) up-regulated genes in the lungs of COVID-19 patients . As SARS-coV-2 infection has a huge impact on the hematopoietic system affecting the myeloid cell maturation, we meta-analyzed the effects of thalidomide and its derivatives on PBMC, bone marrow cells as well as lymphoma cells. Thalidomide and lenalidomide exhibited attenuation of cytokine signaling and inflammation in addition to its anti-angiogenic action (Figure 3A). The drugs affected most of the pathways up-regulated in SARS-coV-2 affected lungs and PBMC (Figure 1A, 3A) in A549 cells, mandating direct investigations in SARS-coV-2 infected models.

COVID-19 coincides with a strong neuro-endocrine modulation because the disease devastates functions of the organs, and naturally the reciprocal communication between the organs of the endocrine stress system gets a set-back . ACE2 is expressed along the hypothalamus, pituitary and adrenal (HPA) axis which is implicated in the stress response and adrenal glands has the highest concentration of virus particles next to lung. A high expression of ACE2 in brain is believed to be the reason for the possible infection of the central nervous system in SARS patients . Chronic elevated stress levels has been reported in SARS and SARS-coV-2 patients even long after the outbreak . Notably, thalidomide is also known for its neuro-endocrine modulation properties. Thalidomide modulates CNS by reducing the generation of pro-inflammatory cytokines such as IL-1, IL-6, IL-8 and TNF- $\alpha$ through NF- $\varkappa$ B inhibition. There was a down-regulation of genes involved in circadian wake cycle (Figure 1B, S2) including PER3 in the PBMC of COVID-19 patients hinting on the possible sleep disturbances in SARS-coV-2 patients. Thalidomide being a well-known antiemetic and sedative action on the neuroendocrine axis would relax the patients which is supported by the report that thalidomide was effective in treating the anxiety and digestive symptoms in the COVID-19 patient .

The anti-inflammatory properties of thalidomide and its analogs through reduction of IL- $1 \beta$, TNF- $\alpha$ expression and NF- $x \mathrm{~B}$ inhibition are well established. SARS-coV-2 infections showing elevated NF- $x$ B signaling and rampage activation of immune response. Unlike other RNA viruses, SARS-coV-2 suppresses TNF receptor-associated factors 3 (TRAF3) activation, inhibiting NF- $\varkappa \mathrm{B}$ and IRFs, leading to suppression of early pro-inflammatory and antiviral responses. Whereas later stages of the infection show an enhanced expression of IRF targets in the lungs with an activation of IL-1, IL-6 and TNF- $\alpha$ expression and inhibition of type I interferon signaling . Activation of IRF and ISRE transcriptional targets in SARS-coV-2 affected lungs is in agreement with previous studies reporting the SARS biology . Thalidomide inhibited LCK activity affecting STAT1 phosphorylation, cytokine mediated signaling, NF- $x$ B signaling, osteoclast 
differentiation and MAPK signaling through modulation of various upstream activators and downstream effectors. Lenalidomide, in addition, suppressed leukocyte differentiation, TLR signaling along with IRF activation in A549 and lymphoma cells. The effects of thalidomide and lenalidomide observed in our study are consistent with the previous studies where thalidomide and lenalidomide has been shown to inhibit IRF and STAT1 phosphorylation resulting in the downregulation of interferon expression and TLR signaling .

The expression profile of SARS-coV-2 infected lungs, PBMC as well as A549 cells show resemblance with profiles of lymphoma, multiple myeloma and SLE. Therefore, drugs which are effective in treating SLE, lymphoma and multiple myeloma might be effective against SARS-coV-2 infection. Thalidomide and its derivatives show impressive efficacy in treating multiple myeloma and certain forms of lymphoma . Remarkably, hydroxychloroquine, an FDA approved SLE drug is currently being used in the management of critically ill SARS-coV-2 patients. CC-220, another thalidomide analog shows very promising results in phase I/II clinical trials against SLE . CC-220 through suppression of Ikaros and Aiolos expression , transcription factors which are essential for differentiation of leukocyte and NK cells thus modulating the innate immune system. As innate immune system pathways are deregulated in SARS-coV-2 infected lung and PBMC, further studies are warranted to investigate the efficacy and safety of CC-220 in treating COVID-19

Any treatment strategy with thalidomide and its analogs including repurposing thalidomide for COVID19 , should consider thalidomide-induced adverse effects including neuropathy and venous thromboembolism (VTE) . There have been many reports on COVID-19 patients develop blood clots, a dangerous issue which might be aggravated with the use of thalidomide and lenalidomide. In addition, lenalidomide might cause cytokine release syndrome in chronic lymphocytic leukemia patient . Therefore, a very careful dosage regimen has to be followed with all these drugs as serious adverse effects have been observed during dose escalation earlier.

\section{Conclusion}

Our study sheds light on the possible mechanisms through which thalidomide and lenalidomide might be effective in the management of SARS-coV-2 pathology. Thalidomide and derivatives effectively modulating various aberrantly regulated pathways infections with abundant pharmacological information available make them promising candidates for the treatment of novel coronavirus infections.

\section{References}

\section{Figure legends}

Figure 1: Characteristics of SARS-coV-2 infected lungs and A549 cells. Gene Set Enrichment Analysis (GSEA) of genes modulated in A) SARS-coV-2 infected lung and B) A549 cells. C) Transcription factor enrichment of genes up-regulated in SARS-coV-2 infection. D) Genes activated in SARS-coV-2 overlapping with genes down-regulated upon kinase perturbations. Up-regulated genes mapped to their transcription factors and kinases in E) SARS-coV-2 lung and F) A549 cells.

Figure 2: Effect of Thalidomide and Lenalidomide on Immune System. GSEA network of gene expression profiles of A) thalidomide and B) lenalidomide treated A549 cells. C) Activities of kinase involved in immunomodulation and MAPK signaling in the presence of thalidomide D) Biological Pathways enriched by kinases affected by thalidomide.

Figure 3: A) GO Biological Process enrichment comparison of gene expression signatures B) SARS-coV-2 infected tissues B) Overexpression of immune response genes in SARS-coV-2 tissues and suppression by thalidomide and lenalidomide C) B cell receptor signaling activated in PBMC of SARS-coV-2 patient and T cell activation in SARS-coV-2 lungs D) Modulation of ERK1 and ERK2 cascade in SARS-coV2 lung and thalidomide in A549.

Figure 4: Activation of A) inflammation, B) TLR signaling, C) NOD-like signaling, D) Osteoblast differentiation and E) Cytokine signaling in SARS-coV-2 and genes targeted by thalidomide and lenalidomide in the signaling pathways. 
Figure 5: Viral life cycle and defense response to virus. A) Up-regulation of pathways pertaining to viral entry, life cycle and antiviral response was observed in SARS-coV-2 infected lungs, PBMC, BALF and A549 cells and their modulation by thalidomide and lenalidomide. Overexpression of genes implicated in B) Interferon signaling and C) Type I interferon signaling in SARS-coV-2 infected lung and A549.

Figure 6: A) Similarity of SARS-coV-2 signatures with disease-specific phenotypes. Differentially modulated genes in SARS-coV-2 and thalidomide treatment similar to profiles of B) lymphoma and D) Multiple Myeloma. C) Overlapping of SARS-coV-2 expression profile of SARS-coV-2 affected lung with systemic lupus erythematosus.
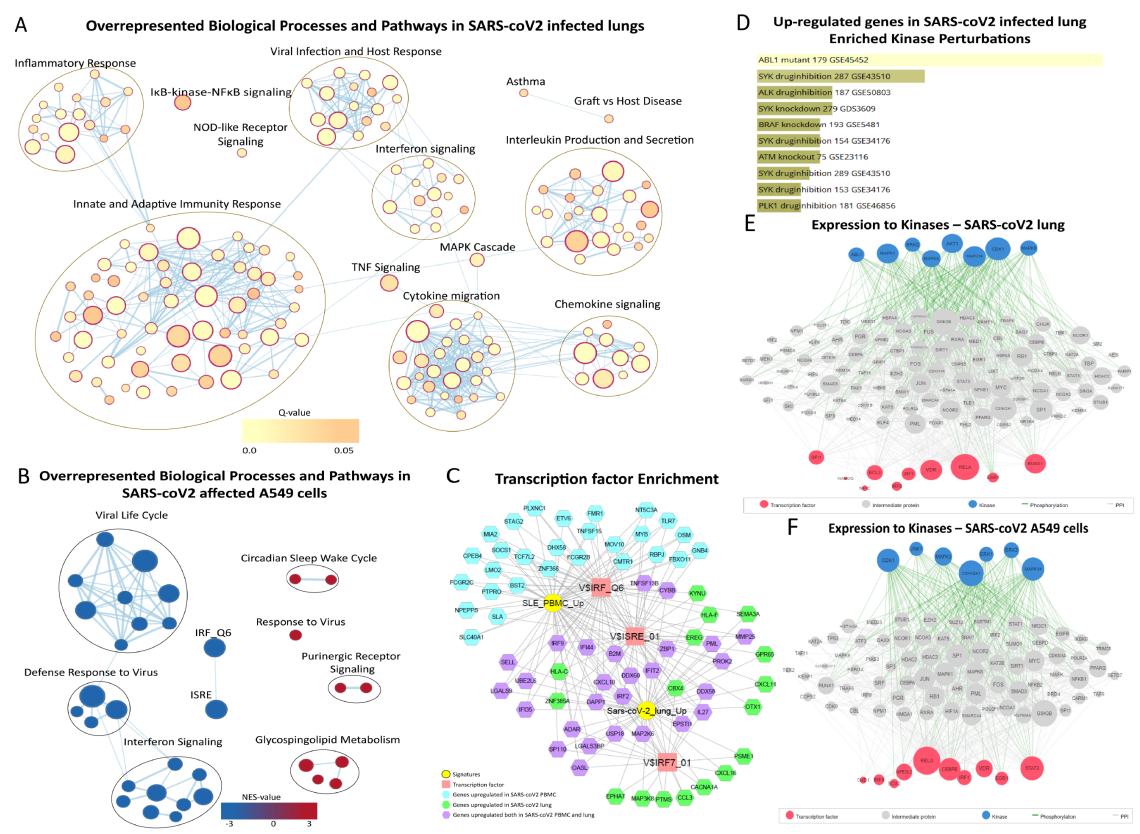
Effect of Thalidomide and Lenalidomide Treatment in A549 cells
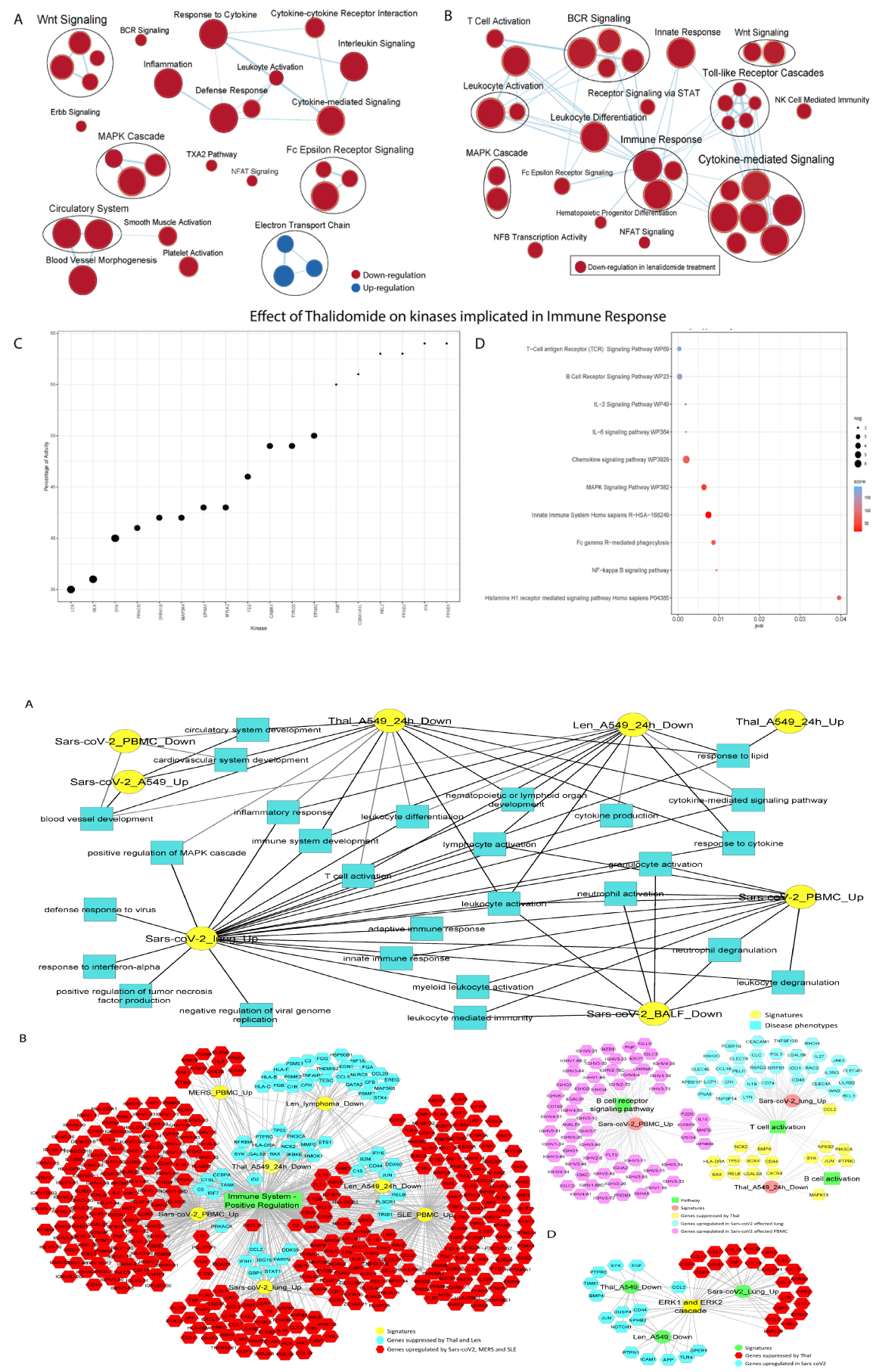

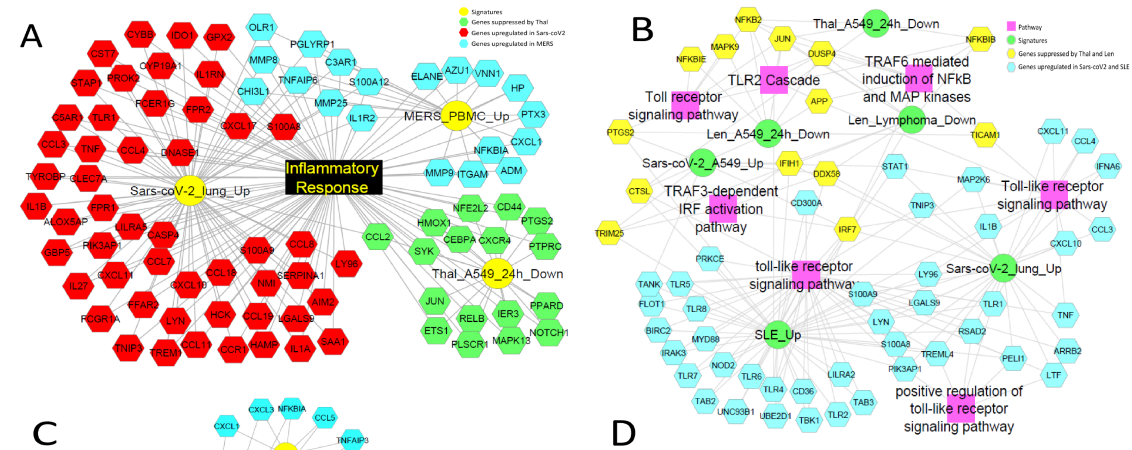

C

$\sum_{10}$

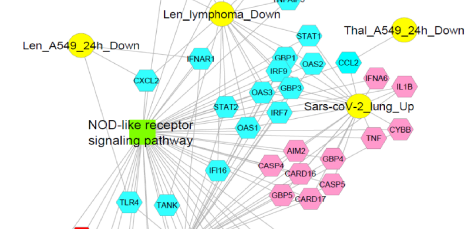

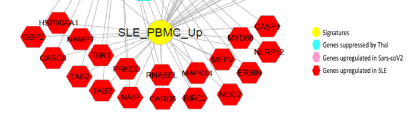

E

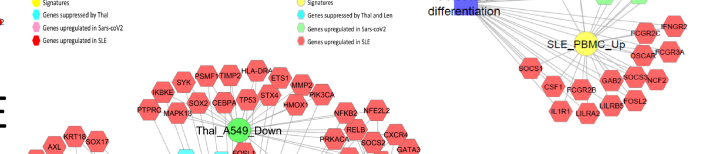

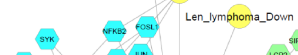

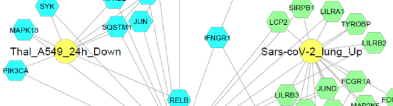

$-1 \times-1$

…

ste.enc-uo
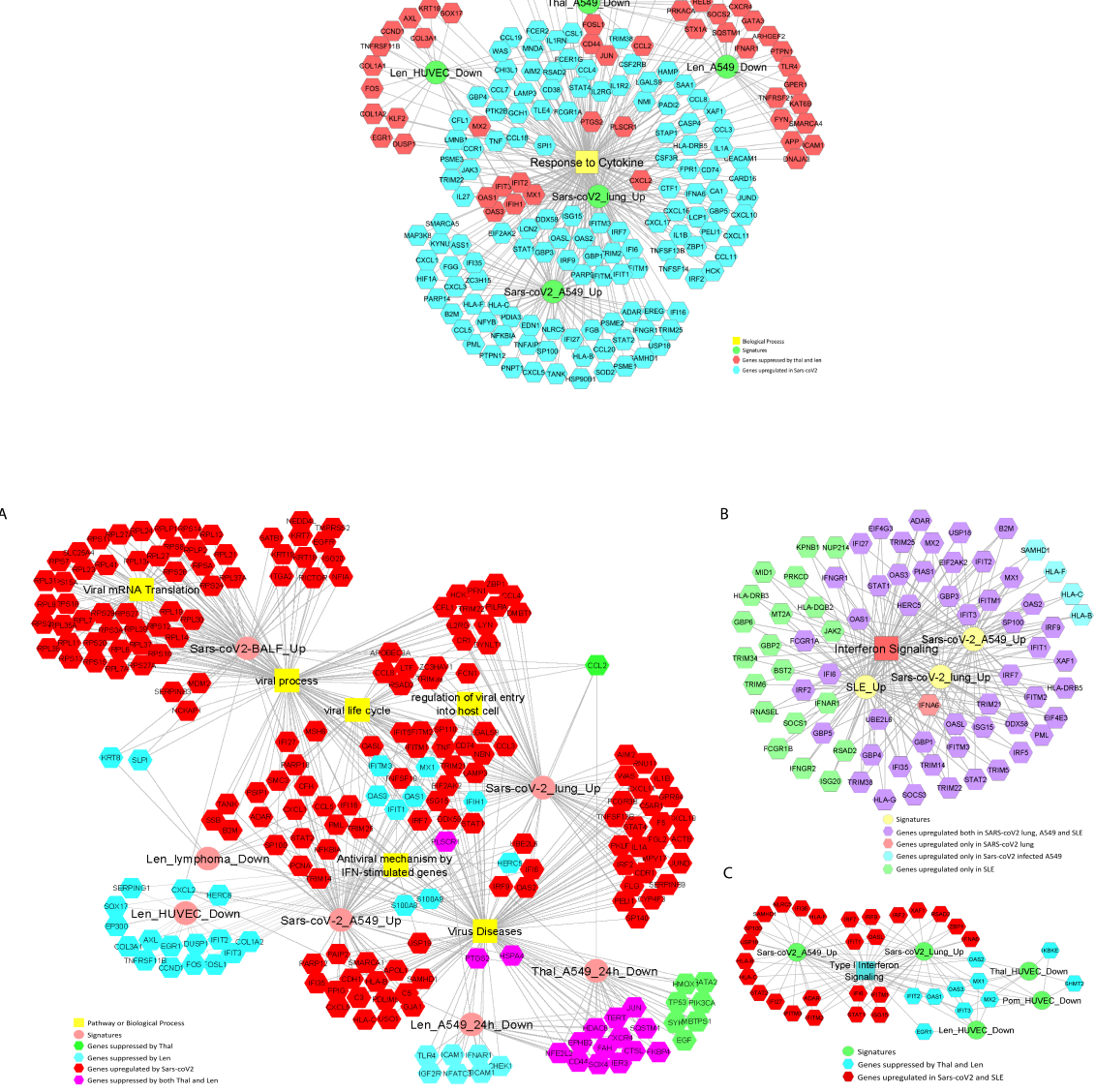

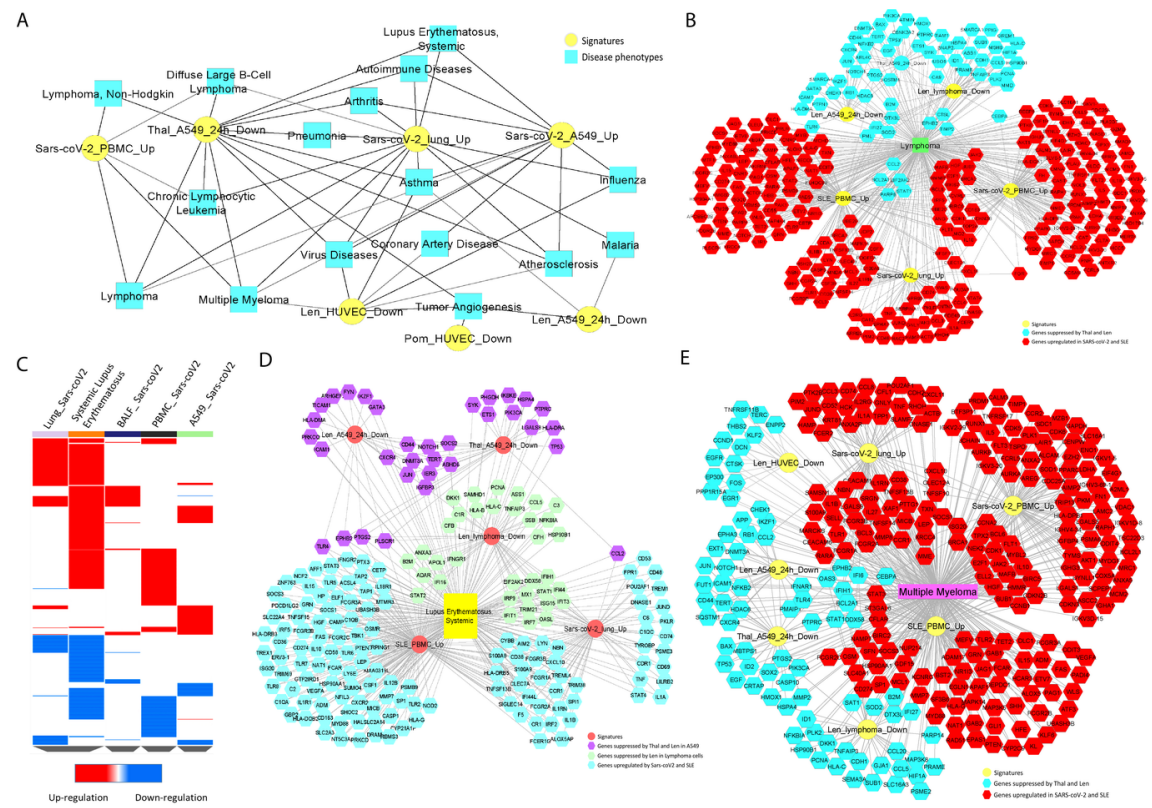